The Effect of Amorphization Conditions on the Measured Activation of Source Drain Extension Implants

Jonathan England, Alexander Kontos, Anthony Renau, Russell Gwilliam, Andrew Smith, Andrew Knights, and Amitabh Jain

Citation: AIP Conference Proceedings 1066, 91 (2008); doi: 10.1063/1.3033691

View online: http://dx.doi.org/10.1063/1.3033691

View Table of Contents: http://aip.scitation.org/toc/apc/1066/1

Published by the American Institute of Physics 


\title{
The Effect of Amorphization Conditions on the Measured Activation of Source Drain Extension Implants
}

\author{
Jonathan England ${ }^{1}$, Alexander Kontos ${ }^{1}$, Anthony Renau ${ }^{1}$, Russell Gwilliam², \\ Andrew Smith ${ }^{2}$, Andrew Knights ${ }^{2}$ and Amitabh Jain ${ }^{3}$ \\ ${ }^{1}$ Varian Semiconductor Equipment, 35 Dory Road, Gloucester, MA 01930, USA \\ ${ }^{2}$ Surrey Ion Beam Centre, Nodus Laboratory, The University of Surrey, Guildford, Surrey. GU2 7XH, UK \\ ${ }^{3}$ External Development \& Manufacturing, Texas Instruments, 13121 TI Boulevard, MS 365, Dallas, TX 75243, USA
}

\begin{abstract}
Un-patterned wafers were processed using low-dose Indium or medium-dose Germanium pre-amorphization implants (PAI) followed by p-type dopant implants of $\mathrm{BF}_{2}$ or carborane $(\mathrm{CBH})$. The wafers were then annealed by RTA (spike), laser anneal (LSA) or combination of LSA and spike. Active dopant distributions calculated from SIMS and sheet resistance measurements compared favorably with those determined by differential Hall, which is a challenging technique for shallow profiles. The trends in $\mathrm{B}$ diffusion behavior and activation are discussed in relation to the different implant damage budgets, damage evolution during the anneals and presence of fluorine. In particular, for low thermal budget LSA only anneals, $\mathrm{CBH}$ implants appear to give higher activation than $\mathrm{BF}_{2}$ due to the absence of fluorine.
\end{abstract}

Keywords: Pre-amorphization, ion implantation, transient enhanced diffusion, activation, laser annealing PACS: 61.72.uf; 85.40.Ry

\section{INTRODUCTION}

Many schemes have been proposed for the production worthy manufacture of shallow, high conductivity, low leakage $32 \mathrm{~nm}$ transistor junctions. The most challenging step of these schemes often involves formation of the PMOS source drain extension. This study compared two PAI schemes previously used at larger design rules, $\mathrm{BF}_{2}$ and orthocarborane $(\mathrm{CBH}[1])$ doping implants and various anneal strategies. Co-implants were not included. The use of the differential Hall (DH) technique for shallow junction metrology was also evaluated.

\section{EXPERIMENTAL CONDITIONS}

All implants were carried out into $200 \mathrm{~mm} \mathrm{Si}$ wafers at Varian using a VIISta HC implanter except for the Indium implants, which were carried out on a VIISta 810 medium current tool at Texas Instruments. PAI conditions of $\mathrm{Ge} / 20 \mathrm{keV} / 3.5 \mathrm{E} 14 / \mathrm{cm}^{2}$ or $\mathrm{In} / 15 \mathrm{keV} / 3 \mathrm{E} 13 / \mathrm{cm}^{2}$ were followed by dopant implants at $0^{\circ}$ tilt of $\mathrm{CBH} / 6.8 \mathrm{keV} / 1.4 \mathrm{E} 14 / \mathrm{cm}^{2}$, or $\mathrm{BF}_{2} / 2 \mathrm{keV} / 1.4 \mathrm{E} 15 / \mathrm{cm}^{2}$. It was noted (see Figure 2) that the $\mathrm{B}$ energies were not exactly matched for these conditions. The $\mathrm{B}$ from the $\mathrm{CBH}$ had a nominal equivalent energy of $500 \mathrm{eV}$ whereas the $\mathrm{B}$ from the $\mathrm{BF}_{2}$ was shallower, with an equivalent energy approximately $10 \%$ lower. The different combinations of implants were annealed with one of the following schemes: LSA at $1270^{\circ} \mathrm{C}$ for $0.8 \mathrm{~ms}$; spike at $950^{\circ} \mathrm{C}$ for $\sim 2$ seconds; LSA followed by a spike (both with the same parameters as above). The sheet resistance, Rs, of each wafer was then measured by a KLATencor RS100 4 point probe.

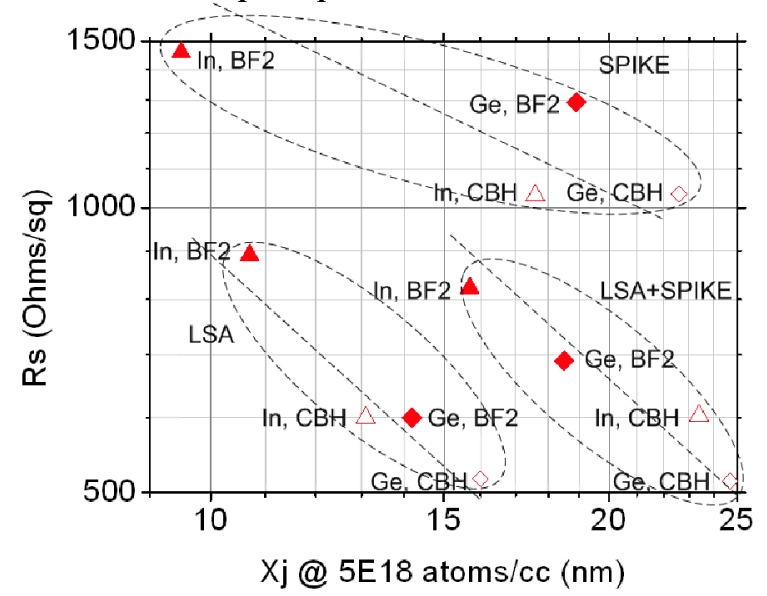

FIGURE 1. An Rs-Xj plot for the PAI, dopant combinations of this study. Ellipses enclose same anneal conditions; dotted lines are guides to the Rs-Xj trends. 
TABLE 1. Comparison of $\mathrm{CBH}$ and $\mathrm{BF}_{2}$ observations

\begin{tabular}{llll}
\hline \multicolumn{1}{c}{ Anneal } & Activation & Diffusion & Mobility \\
\hline Spike & Similar & CBH deeper & Similar \\
LSA & CBH higher & Similar & Similar \\
LSA+ Spike & Similar & CBH deeper & Similar \\
\hline
\end{tabular}

Next, pieces of each wafer were profiled by SIMS, which was carried out at Texas Instruments on a Cameca 6F with $500 \mathrm{eV} \mathrm{O}_{2}{ }^{+}$ions and oxygen leak for boron. The B SIMS profiles for the $\mathrm{CBH}$ implants shows ${ }^{10} \mathrm{~B}+{ }^{11} \mathrm{~B}$, whilst the $\mathrm{BF}_{2}$ shows ${ }^{11} \mathrm{~B}$ only. In this study, the junction depth $(\mathrm{Xj})$ was defined as the depth at which the $B$ concentration was $5 \times 10^{18}$ atoms $/ \mathrm{cm}^{3}$. The Rs-Xj plots for the various samples are shown in Figure 1 and the SIMS profiles are in Figure 2.

The SIMS and Rs data could be combined to estimate the maximum concentration of electrically active boron in the following way. It was assumed that at low concentrations, the activated profile was same as the SIMS profile. However, near the surface where the chemical concentration was high, the active boron concentration was assumed to reach a maximum value. Using the hole mobility as a function of active boron concentration given by Schroder[2], the contribution to the sheet resistance of each part of the boron depth profile could be calculated. The maximum active boron concentration was chosen so that the calculated value of the total sheet resistance matched the measured value. The effects of surface oxide were ignored. The estimated maximum activation concentrations and their associated hole mobilities are reported in Figure 3. The activation levels calculated from SIMS/Rs often agreed with another qualitative estimate of the active $B$ concentration, which was indicated in the SIMS profile where the diffusion tail breaks from the immobile, surface peak[3].

Further pieces of each wafer had van der Pauw structures fabricated on them at The University of Surrey, and DH measurements were carried out[4]. This technique makes repeated Hall and resistivity measurements after thin layers of the material have been removed. In this way, the depth profiles of the electrically activated boron concentrations and mobility could be measured. The Hall scattering factor (the ratio of field-free mobility to that in a magnetic field) has been taken as unity. The technique is limited for shallow junctions by the accuracy of the material removal step. Also, depletion effects mean that the junction is distorted and can only be measured to a limited depth. For these reasons, the results are semi-quantitative but certainly indicative of trends.

Finally, junction leakage measurements were made on mesa diode structures of varying sizes fabricated on other pieces of each wafer at The University of Surrey. This is a more sensitive technique than assessing damage by cross sectional or planar TEM. The junction leakages for all the samples of this study were measured to be extremely small $\left(\sim 10^{-8} \mathrm{~A} / \mathrm{cm}^{2}\right)$.

\section{RESULTS AND DISCUSSION}

The trends in the DH and SIMS/Rs results for activation and mobility (Figure 3) follow each other quite closely. Indeed, applying a reasonable Hall scattering factor of $\sim 0.6$ would bring the maximum activated $\mathrm{B}$ and mobilities into good agreement. The DH would still suggest higher activation (and lower mobility) for the LSA cases than the SIMS/Rs calculations. In most cases, the relative depths of the $\mathrm{DH}$ profiles for the various conditions in Figure 2 agree with the relative depths of the SIMS profiles. Hence DH was a useful technique for this study.

The total damage remaining at the completion of the implants, how this evolved during the various anneals and the presence of fluorine is an important framework within which to explain the observations of diffusion and activation behavior in this study.

When comparing the results for the two PAI conditions on the Rs-Xj plot (Figure 1), it can be seen that Ge always showed lower Rs and deeper $\mathrm{Xj}$ than In. TEM data confirms the TRIM prediction that the In PAI creates only a little damage, located close to the surface. The more complete and deeper damage created by Ge PAI released more interstitials from the EOR damage during the anneals. This may explain the observation of greater B diffusion after Ge, compared to In PAI. The activation measurements (Figure 3) suggest that the lower Rs values for Ge were a consequence of the deeper dopant profiles, rather than higher peak $B$ activation.

TABLE 2. Comparison of observations for the various anneal conditions

\begin{tabular}{lllll}
\hline \multicolumn{1}{c}{ Anneal } & \multicolumn{1}{c}{$\begin{array}{c}\text { B Tail and F Out- } \\
\text { Diffusion }\end{array}$} & EOR Decoration by F & Activation & \multicolumn{1}{c}{$\begin{array}{c}\text { Same Rs-Xj trend for } \\
\text { In \& Ge }\end{array}$} \\
\hline Spike & High TED & No & Low & No \\
LSA & Low TED & Yes & High & Yes \\
LSA + Spike & Intermediate CED & No & High & Yes \\
\hline
\end{tabular}



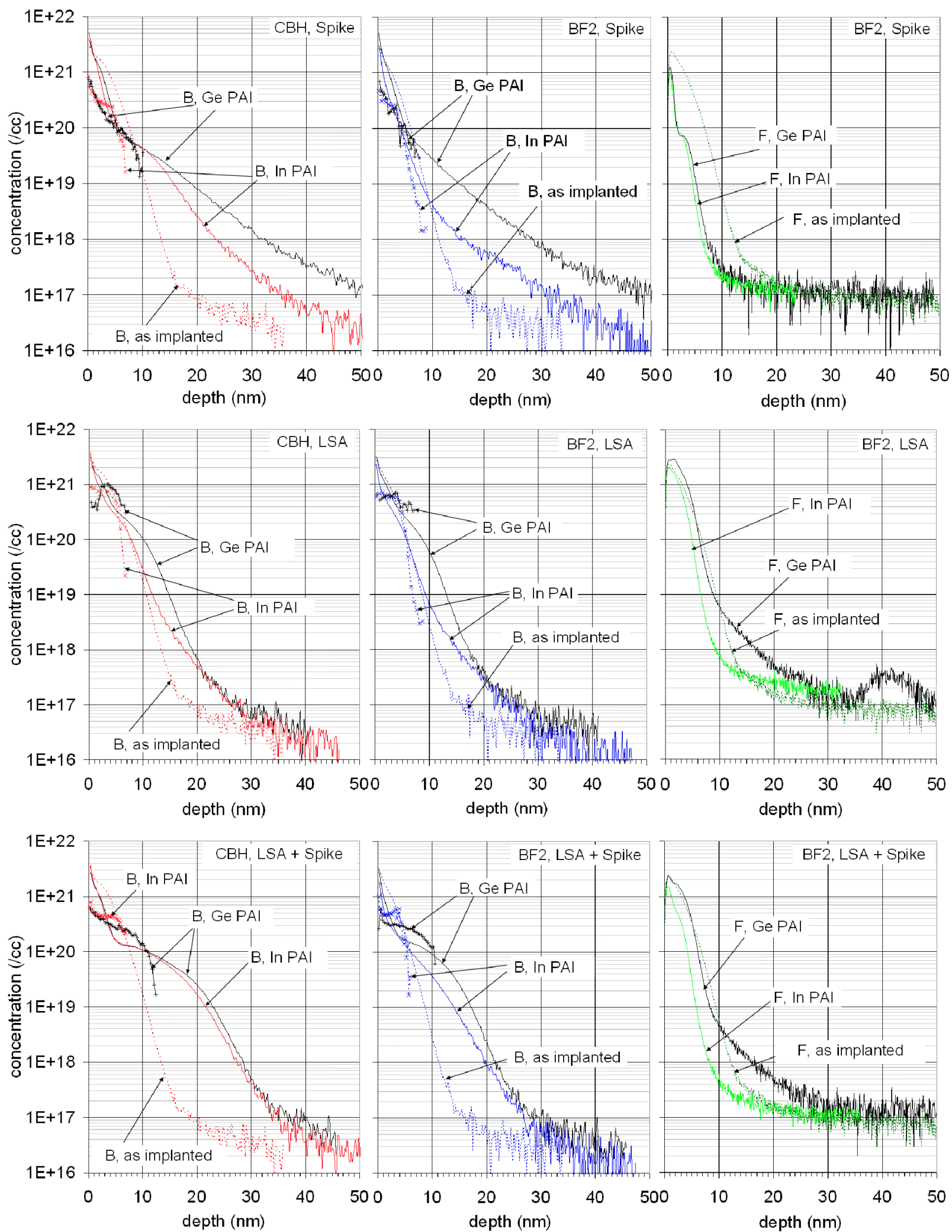

FIGURE 2. SIMS profiles (lines only) before and after annealing and differential Hall profiles (lines and symbols) after annealing for: $\mathrm{B}$ from $\mathrm{CBH} ; \mathrm{B}$ from $\mathrm{BF}_{2} ; \mathrm{F}$ from $\mathrm{BF}_{2}$ implants. 
The effects of the different dopant species are summarized in Table 1 . The fact that different amounts of diffusion can be observed after $\mathrm{BF}_{2}$ and $\mathrm{CBH}$ implants may be a consequence of the fluorine introduced by the $\mathrm{BF}_{2} . \mathrm{F}$ is known to annihilate interstitials, which mediate B diffusion, through its ability to getter vacancies[3]. Other experiments suggest that the intrinsic $\mathrm{C}$ implanted as part of the $\mathrm{CBH}$ molecular implants has little effect on diffusion[1]. (In contrast, when the $\mathrm{C}$ is implanted separately, the deeper $\mathrm{C}$ impedes the EOR interstitial flux from interacting with the shallow $\mathrm{B}[5]$ ). In addition, the $\mathrm{CBH}$ would be expected to retain more damage after implant than $\mathrm{BF}_{2}$ due to the molecular effect[6] (and the CBH energy was also slightly higher). For the In PAI case, where the dopant damage was a significant addition to the total damage budget, this mechanism may account for the larger difference in $\mathrm{Xj}$ between $\mathrm{CBH}$ and $\mathrm{BF}_{2}$, compared to the Ge case, where the dopant damage was swamped by that from the Ge.

The effects of the anneal conditions are summarized in Table 2. The observations of the SIMS profiles are consistent with the spike anneal promoting the greatest amount of damage evolution and interstitial release, leading to the deep tails indicative of transient enhanced diffusion (TED). In contrast the LSA evolved the damage such that few interstitials were released and the profiles remained shallow. The spike after the LSA appears to not have been able to release as many interstitials as the spike alone, as there is little further TED in the tail. However, the profiles become boxier as concentration enhanced diffusion (CED), driven by the boron activated during the LSA step, occurs.

The clearest trends in the activation measurements (Figure 3) are that the LSA gave much higher activation than either spike or LSA + spike, and that $\mathrm{CBH}$ showed higher activation than $\mathrm{BF}_{2}$ after the LSA anneals. As the formation of Boron Interstitial Clusters (BICs) is an important mechanism in the deactivation of $\mathrm{B}$, the first observation is consistent with the explanation again that the LSA released the lowest number of interstitials for BIC formation. The difference in activation for the dopant species after LSA may be explained by a lower competition for lattice sites between $\mathrm{B}$ and $\mathrm{C}$ after $\mathrm{CBH}$ implants compared to that between $\mathrm{F}$ and $\mathrm{B}$ after $\mathrm{BF}_{2}$. There is a hint that $\mathrm{CBH}$ may have had slightly lower activation than $\mathrm{BF}_{2}$ for the In PAI cases that were followed by spike and LSA + spike anneals. Similar to the diffusion argument, this may be explained by the dopant damage being a large fraction of the total damage budget (and the total damage was more surface peaked) for the In PAI so that the spike anneals may have allowed more extensive BIC formation after $\mathrm{CBH}$ than $\mathrm{BF}_{2}$. After Ge PAI, the presence of $\mathrm{F}$ appears to be a more important factor.

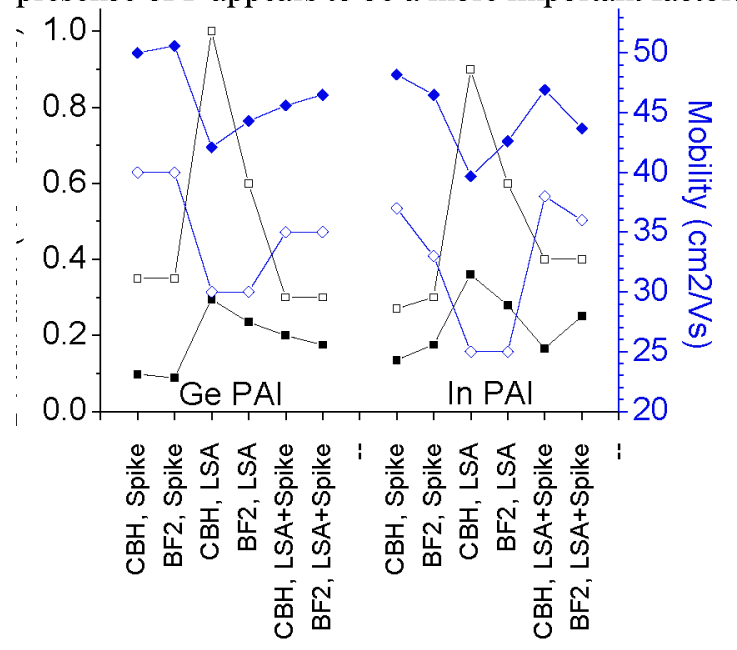

FIGURE 3. Boron activation: (๓) calculated from Rs/SIMS; ( $\square$ ) measured by differential Hall; and mobility: $(\diamond)$ from Rs/SIMS; $(\diamond)$ differential Hall

\section{CONCLUSIONS}

Differential Hall has shown itself to be a useful, semi-quantitative technique for $32 \mathrm{~nm}$ junction metrology. Many of the observations of PAI, dopant and anneal schemes could be explained by considering implant damage, thermal budgets and the presence of fluorine. In particular, the higher levels of boron activation after laser anneals for $\mathrm{CBH}$ compared to $\mathrm{BF}_{2}$ implants, regardless of PAI scheme, appears to be attributed to the absence of fluorine, rather than damage considerations.

\section{REFERENCES}

1. A. Renau, Extended Abstracts of the Seventh International Workshop on Junction Technology, pp. 107-116 (2007)

1. D. Schroder, "Semiconductor Materials and Device Characterization" (2nd ed) John Wiley and Son, New York 1998, pp. 551-553

2. N. Cowern, B. Colombeau et al., Appl. Phys. Lett. 86, 101905 (2005)

4. N. Bennett, A. Smith, B. Colombeau, R. Gwilliam, N. Cowern, B. Sealy, Mat. Sci. and Eng. B 124-125, 305$309(2005)$

5. E. Collart, D. Kirkwood et al., Proceeding of IIT2004, Part II, 11-14 (2005)

6. L. Perlaz, L.A. Marques and J. Barbolla, J. Appl. Phys. 96, 5947-5976 (2004) 